\title{
Spatial Intratumoral Heterogeneity Expression of PD-L1 Antigen in Head and Neck Squamous Cell Carcinoma
}

\author{
Nir Hirshoren ${ }^{a}$ Issa Al-Kharouf ${ }^{b}$ Jeffrey M. Weinberger ${ }^{a}$ Ron Eliashara \\ Aron Popovtzer ${ }^{c}$ Ariela Knaanie ${ }^{a}$ Yakov Fellig $^{b}$ Tzahi Neuman $^{b}$ Karen Meir $^{b}$ \\ Alexander Maly ${ }^{b}$ Gilad W. Vainer ${ }^{b}$ \\ ${ }^{a}$ Department of Otolaryngology/Head and Neck Surgery, Hadassah Hebrew University Medical Center, Jerusalem, \\ Israel; 'bepartment of Pathology, Hadassah Hebrew University Medical Center, Jerusalem, Israel; 'Sharett Institute \\ of Oncology, Hadassah Hebrew University Medical Center, Jerusalem, Israel
}

\section{Keywords}

Programmed cell death ligand $1 \cdot$ Head and neck neoplasm · Survival analysis . Tissue array analysis

\begin{abstract}
Introduction: Immune-checkpoint inhibitors have demonstrated a significant survival benefit in metastatic and nonresectable head and neck squamous cell carcinoma (HNSCC). Patients with a combined positivity score (CPS) of 20 and higher benefit the most from therapy. Inaccurate definition of the CPS category might lead to the incorrect stratification of patients to immunotherapy. This study's main aim was to investigate programmed death-ligand 1 (PD-L1) antigen expression in HNSCC in diverse clinical situations and histological settings. Materials and Methods: This is a prospective cohort study conducted in a tertiary referral medical center. Tissues were investigated for PD-L1 expression using the FDA-approved 22C3 immunohistochemistry assay (Dako). We analyzed potential associations between the CPS category and meaningful demographic, clinical, and outcome metrics. Furthermore, we investigated morphologi-
\end{abstract}

cally separate sites for CPS scores in whole surgical tissue specimens and matched preoperative biopsies. Results: We analyzed 36 patients, of whom 26 had oral cavity SCC and 10 had laryngeal SCC. The overall, disease-specific, and progression-free survival of the HNSCC group of patients were not associated with the CPS category $(p=0.45, p=0.31$, and $p=0.88$, respectively). There was a significant $(18 \%, 95 \% \mathrm{Cl}$ $0.65-0.9$ ) inconsistency between the CPS category determined in biopsies versus whole carcinoma analyses. We also found an uneven distribution of whole-tumor CPS attributed to spatial carcinoma invasiveness, tumor differentiation, and inflammatory cell infiltration heterogeneity. Discussion and Conclusions: Our data suggest that careful selection of tumor area for CPS analysis is important. PD-L1 antigen expression, clinically represented by CPS, may be up- or down-categorized in different clinical and pathological circumstances. The high whole-tissue CPS category scatter may clinically result in potential treatment modifications. We argue that CPS analysis requires not only adequacy (at least 100 viable tumor cells), but also correct representation of the tumor microenvironment.

(c) 2021 The Author(s)

Published by S. Karger AG, Basel
(C) 2021 The Author(s)

Published by S. Karger AG, Basel

This is an Open Access article licensed under the Creative Commons Attribution-NonCommercial-4.0 International License (CC BY-NC) (http://www.karger.com/Services/OpenAccessLicense), applicable to the online version of the article only. Usage and distribution for commercial purposes requires written permission.
Correspondence to:

Nir Hirshoren, drnir@ hadassah.org.il 


\section{Introduction}

Programmed death-ligand 1 (PD-L1) acts as a coinhibitory immune system factor. PD-L1 in tumor cells bind to the programmed death-1 (PD-1) receptor on cytotoxic $\mathrm{T}$ lymphocytes leading to negative regulatory signals and loss of anti-tumor activity [1]. Blocking antibodies of PD-1 introduced a revolutionary change [2] in the clinical approach to metastatic and non-resectable aerodigestive head and neck squamous cell carcinoma (HNSCC) $[3,4]$. These antibodies restore the cytotoxic antitumor function of $\mathrm{T}$ lymphocytes and elicit a significant clinical response [5]. Unfortunately, only a subset of tumors respond to this therapy. It is vital to establish improved criteria to better select patients who would benefit from checkpoint inhibitors.

$\mathrm{PD}-\mathrm{L} 1$ expression is a potential predictive biomarker of the response to anti-PD-L1/anti-PD-1 therapy in multiple cancers, including HNSCC. Improved survival was associated with a higher combined positivity score (CPS) to such an extent that immunotherapy by itself was significantly effective as first-line treatment in metastatic and non-resectable cases of HNSCC if CPS was 20 or more [6]. Several research groups demonstrated a possible association between PD-L1 intensity and various demographic data, clinical severity, and survival metrics. Moreover, different histopathological scenarios, including heterogeneity of PD-L1 expression both within and between tumor sites, is a well-documented phenomenon. All of the above may compromise the predictive power of PD-L1 and the current literature in this regard is inconsistent [7], precluding firm conclusions. Our main hypothesis is that heterogenic PD-L1 tissue expression in HNSCC may attribute to CPS category mismatch in biopsies versus whole carcinoma analyses, affecting treatment decisions.

\section{Methods}

\section{Patients and Study Design}

This study is a prospective analysis of HNSCC patients treated at a tertiary referral medical center. The study was approved by the Institutional Review Board (0346-12-HMO). All patients signed an informed consent form following a detailed explanation. Data anonymization was rigorously maintained.

\section{Primary Endpoints}

A comparative analysis was made of PD-L1 antigen expression in preoperative biopsies versus samples taken from separate distinct sites within the excised tumor. Overall, disease-free, and progression-free survival (OS, DFS, and PFS) were determined for various PD-L1 antigen expression levels (CPS category) and compared with survival metrics.

PD-L1 Antigen Expression Heterogeneity
Patients

Consecutive patients with HNSCC treated at Hadassah Medical Center between June 1, 2016 and June 1, 2019 were recruited. We excluded patients with less than 6 months of follow-up and those who did not provide signed informed consent. The medical and surgical records of all HNSCC patients included in the study were surveyed. The following baseline parameters were retrieved: age, gender, comorbidities, tumor site and stage (AJCC Staging Manual, 8th edition), and treatment modality (whole primary tumor excision, radiation, and chemotherapy). Dates of diagnosis, disease progression, death, cause of death, and last follow-up were documented for the survival metrics.

Immunohistochemical Staining for PD-L1 Antigen Expression

Archival tissues were retrieved for PD-L1 analysis. The analysis was performed using the FDA-approved 22C3 assay (Dako) [8]. All HE slides were analyzed by several pathologists. If a case had more than a single block, the pathologist deliberately picked areas showing different HE morphologies for CPS analyses. All PD-L1 staining was analyzed by an experienced, well-trained single observer (G.V.) blinded to the relation of the cases to each other and to the clinical data. All cases were considered adequate for interpretation if at least 100 tumor cells were present. CPS divides the number of PD-L1 staining cells (tumor cells, lymphocytes, macrophages) by the total number of viable tumor cells, multiplied by 100 [9].

Each CPS measurement was evaluated at least twice following a "wash-out" period of 1 week. The patient CPS score was determined by the average score of analyses in the whole surgical specimen tissue. CPS levels were analyzed as continuous (0-100) and as three clinical categories. The three PD-L1 clinical expression categories were determined according to accepted CPS ranges in HNSCC: $<1,1$ to $<20$, and $\geq 20$ [6]. We first investigated the correlation between presurgical biopsies and surgical whole specimens. We then investigated surgical whole tissue homo/heterogeneity as we specifically enriched for different morphological samples in this cohort (by HE, blinded to the CPS score), investigating morphological features that may lead to different PD-L1 expression patterns.

\section{Statistical Analysis}

All statistical analyses were performed using SAS software version 9.04 by a statistician. $\chi^{2}$ or Fisher exact tests were used for comparison of qualitative parameters. The Student $t$ test and Mann-Whitney test were used for quantitative parameters and the ANOVA test for the comparison of more than two subgroups. To examine the intratumor heterogeneity, we calculated the percentage of cases in which there was no agreement on the CPS clinical category classification and the corresponding confidence interval. Kaplan-Meier analysis was used for OS, DFS, and PFS estimation. The log-rank test was used to compare the survival curves. A $p$ value $\leq 0.05$ was considered statistically significant.

\section{Results}

\section{Patients and Disease Characteristics}

Overall, we analyzed 121 separate CPS measurements in 65 SCC tissues of 36 patients with head and neck can- 
Table 1. Demographic and clinical data of 36 HNSCC patients

\begin{tabular}{llc}
\hline Cancer origin & $\begin{array}{l}\text { Oral cavity } \\
\text { Larynx }\end{array}$ & $26(72.2)$ \\
\hline Gender & Females & $10(27.8)$ \\
\hline Mgale & & $14(38.9)$ \\
\hline Comorbidities & Comorbidities presence & $22(61.1)$ \\
\hline Smoking habit & No comorbidities & $58.8 \pm 13.1$ \\
\hline & Smoking & $24(66.7)$ \\
Disease stage & No smoking & $12(33.3)$ \\
& Stage 1 & $14(40)$ \\
& Stage 2 & $21(60)$ \\
\hline OS & Stage 3 & $3(8.3)$ \\
SFS & & $8(22.2)$ \\
PFS & 3-year survival 4 & $3(8.3)$ \\
& & $22(61.1)$ \\
\hline
\end{tabular}

Values are presented as $n(\%)$ or as indicated.

Table 2. CPS level and relevant comparison analyses of 36 head and neck SCC patients

\begin{tabular}{llcc}
\hline & & Median CPS (IQR) & $p$ value \\
\hline Cancer origin & Oral cavity & $8.8(4.3-8.8)$ & $0.001^{*}$ \\
& Larynx & $0.8(0.3-2.8)$ & $0.017^{*}$ \\
\hline Gender & Female & $14.9(6.3-81.1)$ & $0.0045^{*}$ \\
& Male & $2.7(0.8-22.5)$ & $0.021^{*}$ \\
\hline Comorbidities & Comorbidities presence & $3.6(0.8-8.8)$ & 0.2 \\
& No comorbidities & $30.6(6.1-92.1)$ & \\
\hline Smoking habit & Smoking & $2.4(0.5-6.3)$ & $21(3.8-81.1)$ \\
& No smoking & $38.8(5-100)$ & 0.45 \\
Disease stage & Stage 1 & $9.5(2.4-22.6)$ & 0.31 \\
& Stage 2 & $3.9(0.8-21)$ & 0.88 \\
\hline Stage 3 & Stage 4 & & \\
& OS & & \\
& DFS & & \\
& PFS & & \\
\hline
\end{tabular}

* Indicates a statistically significant difference.

cers. The study included 14 females (38.9\%) and 22 males (61.1\%) with a composite average age of $58.8 \pm 13.1$ years. Patients were treated with curative intent and followed in the surgical oncology clinic for an average of 23.5 months.
Most patients (72.2\%) had comorbidities, mainly diabetes mellitus. Forty percent were cigarette smokers with a median smoking history of 30 pack years (range 1-60). Almost $70 \%$ of patients had advanced-stage disease ac- 
Fig. 1. CPS of 23 cases in pre- and postoperative biopsies (blue and red, respectively). The $y$-axis represents the CPS categories: $0=$ CPS $<1 ; 1=$ CPS 1 to $<20 ; 2=$ CPS $\geq 20$.

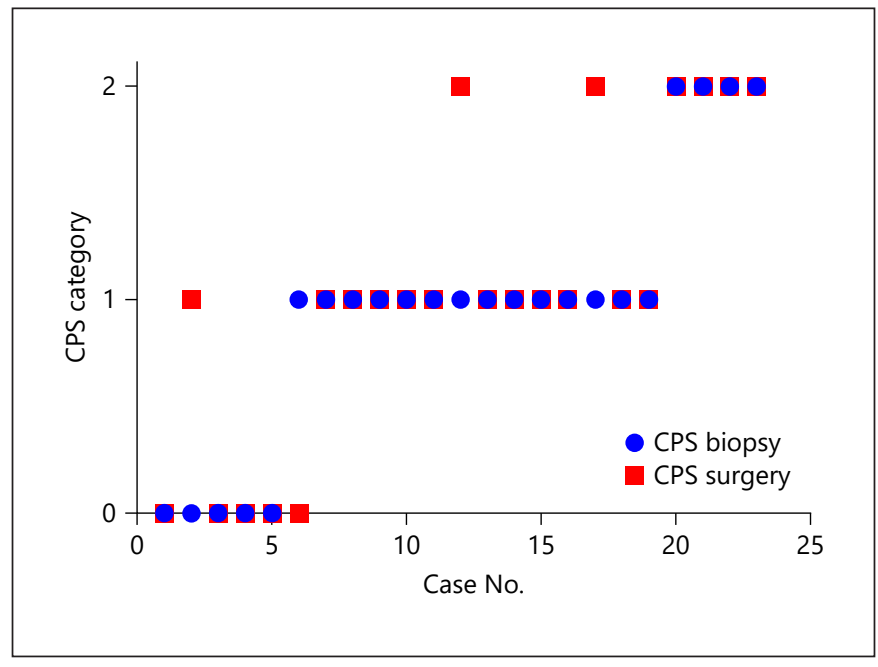

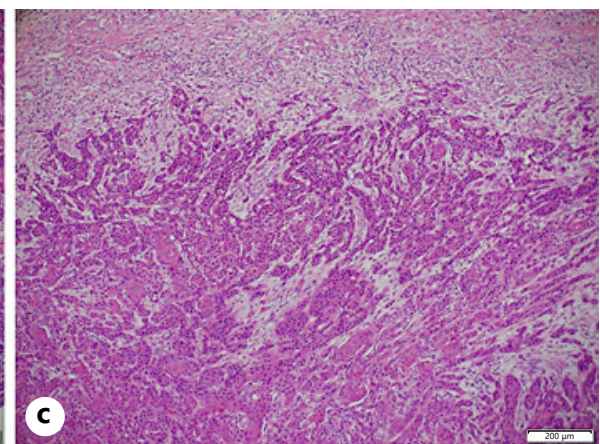
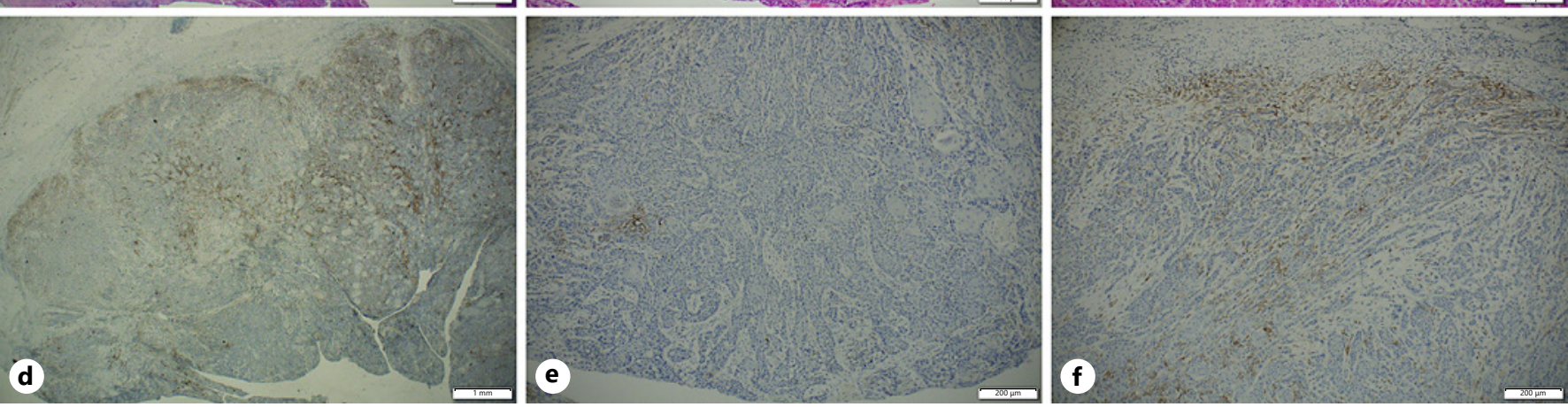

Fig. 2. HE stain $(\mathbf{a}-\mathbf{c})$ and paired FDA-approved $22 \mathrm{C} 3$ assay $(\mathbf{d}-\mathbf{f})$ of a whole surgical tissue. Low magnification (a, d) and higher magnifications of the superficial area $(\mathbf{b}, \mathbf{e})$, and the deeper area $(\mathbf{c}, \mathbf{f})$.

cording to the 8th edition of the AJCC staging system. The 5-year OS, DFS, and PFS were 76\% (95\% CI 56-91\%), $82 \%$ (95\% CI 66-94\%), and 59\% (95\% CI 40-77\%), respectively (Table 1).

\section{CPS Analyses}

Association with Demographic, Tumor Location, Clinical, and Survival Metrics

The median CPS of all patients was $6.1(0-100)$ with a higher score in tongue carcinoma compared to laryngeal carcinoma (median CPS 8.8 vs. $0.8, p=0.0013$ ). 

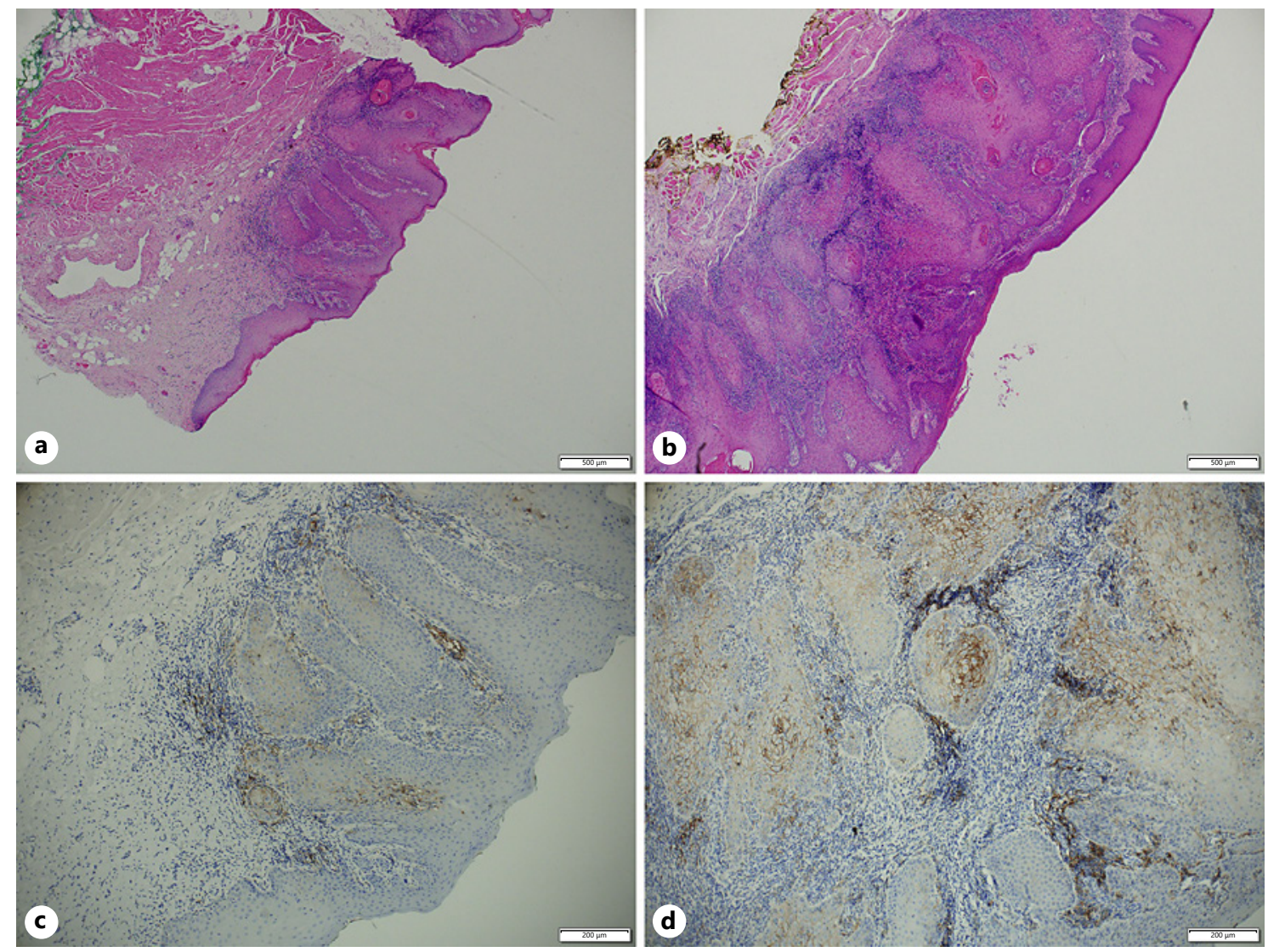

Fig. 3. HE stain (a, b) and paired FDA-approved 22C3 assay (Dako; c, d) of consecutive blocks of biopsy specimen. A region demonstrating smaller invasion and less inflammatory infiltrate $(\mathbf{a}, \mathbf{c})$, when compared with $\mathbf{b}$ and $\mathbf{d}$.

Higher PD-L1 antigen expression was associated with female gender (median CPS 14.9 vs. $2.7, p=0.017$ ), non-smokers (median CPS 21 vs. $2.4, p=0.02$ ), and the absence of comorbidities (median CPS 30.6 vs. $3.6, p=$ 0.0045).

Neither disease stage $(p=0.2)$ nor age had an association with CPS (Pearson correlation coefficient, $r=$ approx. 0.2). The OS, DFS, and PFS of the HNSCC group of patients were not associated with the CPS category ( $p=$ $0.45, p=0.31$, and $p=0.88$, respectively; Table 2 ).

Association with Surgical and Histological

Parameters

Although the numbers are small, we examined 6 tumors and their corresponding lymph node metastases. We found consistent PD-L1 expression between the primary tumor and its metastasis $(p=0.656)$.

We investigated the correlation between the biopsy and corresponding surgical specimens. We had 23 pairs of preoperative biopsies and surgical whole specimens (shown in online suppl. Fig. 1; for all online suppl. material, see www.karger.com/doi/10.1159/000515441), of which 4 (18\%) showed inconsistent scoring (95\% CI $0.65-0.9)$. In 3 cases, higher CPS categories were demonstrated on the surgical whole-tissue analyses. Most CPS category shift occurred when biopsies suggested an intermediate CPS category (Fig. 1). In 19 surgical specimens we highlighted morphological features that may lead to a variable distribution of PD-L1 expression. Several pathologists performed careful histological examination of 5 cases showing significant PD-L1 heterogeneity. We noted that a higher CPS score could be found in: (1) the tumor leading edge (Fig. 2; the deep portion of cancer facing the surrounding healthy tissue); (2) near dense inflammatory cell infiltration (Fig. 3); (3) lower tumor keratinization (Fig. 4), and (4) the largest focus of invasive tumor (Fig. 3). 

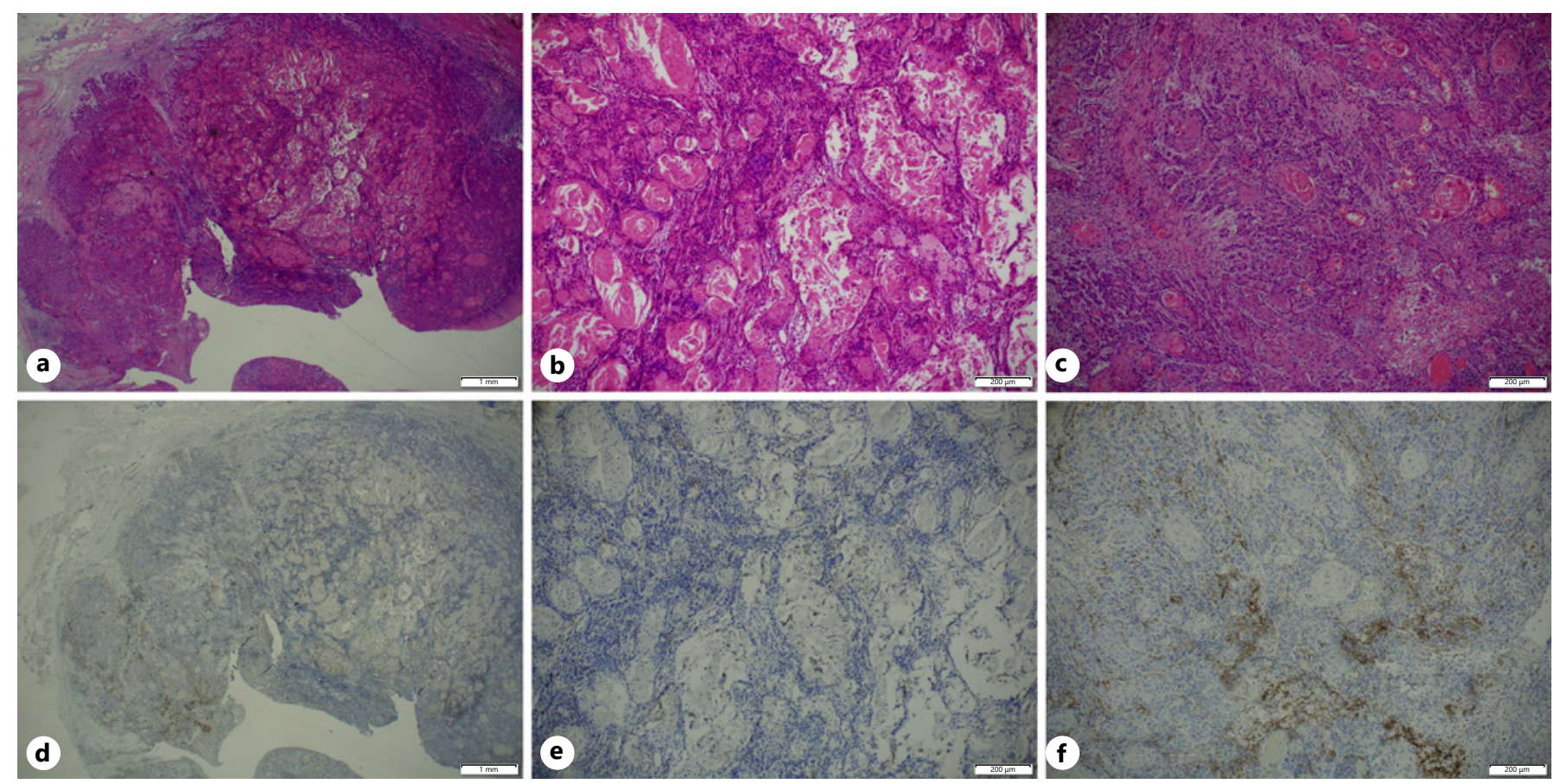

Fig. 4. HE stain (a-c) and paired FDA-approved 22C3 assay (Dako; $\mathbf{d}-\mathbf{f}$ ) of whole surgical tissue. Low magnification $(\mathbf{a}, \mathbf{d})$ and higher magnification $(\mathbf{b}, \mathbf{e})$ of the keratinized area. $\mathbf{c}, \mathbf{f}$ Higher magnification of non-keratinized area.

\section{Discussion}

PD-L1 expression, determined by immunohistochemical staining, is a novel, crucial parameter that predicts HNSCC response to immunotherapy. An appropriate treatment decision in HNSCC is highly correlated with the CPS category. Therefore, emphasis should be placed on clarifying clinical and pathological parameters associated with CPS. The selected area for CPS analysis, representing the tumor immunobiology environment, becomes a significant cause of CPS variability or "scatter."

We did not demonstrate an association between the CPS category and disease stage or survival metrics. Indeed, the role of CPS PD-L1 expression as a prognostic factor is controversial [1]. Carcinomas from other head and neck sites, mostly the oral cavity, oropharynx, and larynx [10], failed to demonstrate survival prediction by PD-L1 expression [11]. Not surprisingly, the disease stage (corresponding with survival) was not associated with PD-L1 expression in most study groups [12].

In accordance with others, we noted a significant association between the absence of smoking [7], female gender, and absence of comorbidities [11]. The relatively rich inflammatory milieu in the tongue (compared to the larynx) may explain the significantly higher CPS we have demonstrated in tongue carcinoma [13].

One of the main findings in our HNSCC study group is CPS category inconsistency when comparing preoperative biopsies to samples from the excised tumor specimens (18\% of cases), and also when comparing different areas within the same excised tumor. These inconsistencies could lead to sharply divergent treatment strategies if based solely on the CPS numerical category. The intratumoral PD-L1 expression spatial heterogeneity may be the cause of the discordance between (randomized) diagnostic biopsies and surgical specimens, which is well known in non-small cell lung cancer [14-16]. Similar results were recently published by another group [17]. Future studies are needed, investigating the actual checkpoint inhibitor response corresponding with CPS scattering. Currently, CPS analysis is considered adequate if at least 100 viable tumor cells are present. Our data suggest that the best reflecting tumor immune-biology environment areas should be selected. These include areas with less keratinization, more invasive component, a deep tumor leading edge, and densely inflamed areas. 


\section{Conclusion}

PD-L1 expression (reported as CPS) is a novel predictor for immunotherapy benefit in metastatic and inoperable head and neck cancer patients. Substratification of the CPS accounting for different locations of the tumor (tongue vs. larynx), gender, smoking habits, and comorbidities may have clinical relevance. Furthermore, our data suggest that careful selection of tumor area for CPS analysis is important. We argue that CPS analysis requires not only adequacy (at least 100 viable tumor cells), but also correct representation of the tumor microenvironment.

\section{Statements of Ethics}

Our research complies with the guidelines for human studies and the research was conducted ethically in accordance with the World Medical Association Declaration of Helsinki. The study was approved by the local Institutional Review Board (0346-12HMO). All patients signed an informed consent form following a detailed explanation. Data anonymization was rigorously maintained.

\section{Conflict of Interest Statement}

G.W.V. reports grants, personal fees, non-financial support, and fees for travel and lecture from MSD, Roche, Pfizer, Abbvie, Takeda, BMS, Bayer, and AstraZeneca; a grant from MSD is directly related to this work. The other authors disclose no conflicts of interest or financial support.

\section{Funding Sources}

This research did not receive any specific grant from funding agencies in the public, commercial, or not-for-profit sectors.

\section{Author Contributions}

N.H. and G.W.V.: conceptualization, analysis, methodology, project administration, supervision, validation, and writing. I.A.K.: conceptualization, data curation, methodology, reviewing, and discussion. J.W.: conceptualization, project administration, supervision, validation, and writing. R.E. and A.P.: conceptualization, methodology, supervision, validation, and review. A.K.: conceptualization, analysis, methodology, validation, and writing. Y.F. and T.N.: analysis, supervision, validation, and review. K.M.: conceptualization, methodology, supervision, validation, and writing. A.M.: analysis, methodology, supervision, and review. All authors gave their agreement for publication.

\section{References}

1 Lenouvel D, González-Moles MÁ, Talbaoui A, Ramos-García P, González-Ruiz L, RuizÁvila I, et al. An update of knowledge on PDL1 in head and neck cancers: physiologic, prognostic and therapeutic perspectives. Oral Dis. 2020;26(3):511-26.

2 Pardoll DM. The blockade of immune checkpoints in cancer immunotherapy. Nat Rev Cancer. 2012;12(4):252-64.

3 Cohen EEW, Soulières D, Le Tourneau C, Dinis J, Licitra L, Ahn MJ, et al. Pembrolizumab versus methotrexate, docetaxel, or cetuximab for recurrent or metastatic head-and-neck squamous cell carcinoma (KEYNOTE-040): a randomised, open-label, phase 3 study. Lancet. 2019;393(10167):156-67.

4 Ferris RL, Blumenschein G, Fayette J, Guigay J, Colevas AD, Licitra L, et al. Nivolumab for recurrent squamous-cell carcinoma of the head and neck. N Engl J Med. 2016;375(19): 1856-67.

5 Le X, Ferrarotto R, Wise-Draper T, Gillison $\mathrm{M}$. Evolving role of immunotherapy in recurrent metastatic head and neck cancer. J Natl Compr Canc Netw. 2020;18(7):899-906.

6 Burtness B, Harrington KJ, Greil R, Soulières D, Tahara M, de Castro G, et al. Pembrolizumab alone or with chemotherapy versus cetuximab with chemotherapy for recurrent or metastatic squamous cell carcinoma of the head and neck (KEYNOTE-048): a ran- domised, open-label, phase 3 study. Lancet. 2019;394(10212):1915-28.

7 Ahmadi N, Gao K, Chia N, Kwon MS, Palme CE, Gupta R, et al. Association of PD-L1 expression in oral squamous cell carcinoma with smoking, sex, and p53 expression. Oral Surg Oral Med Oral Pathol Oral Radiol. 2019; 128(6):631-8.

8 Roach C, Zhang N, Corigliano E, Jansson M, Toland G, Ponto G, et al. Development of a companion diagnostic PD-L1 immunohistochemistry assay for pembrolizumab therapy in non-small-cell lung cancer. Appl Immunohistochem Mol Morphol. 2016 Jul;24(6):3927.

9 Outh-Gauer S, Morini A, Tartour E, Lépine C, Jung AC, Badoual C. The microenvironment of head and neck cancers: papillomavirus involvement and potential impact of immunomodulatory treatments. Head Neck Pathol. 2020;14(2):330-40.

10 Pai SI, Cohen EEW, Lin D, Fountzilas G, Kim ES, Mehlhorn H, et al. SUPREME-HN: a retrospective biomarker study assessing the prognostic value of $\mathrm{PD}-\mathrm{L} 1$ expression in patients with recurrent and/or metastatic squamous cell carcinoma of the head and neck. J Transl Med. 2019;17(1):429.

11 Yang WF, Wong MCM, Thomson PJ, Li KY, $\mathrm{Su}$ YX. The prognostic role of PD-L1 expression for survival in head and neck squamous cell carcinoma: a systematic review and metaanalysis. Oral Oncol. 2018;86:81-90.

12 Ukpo OC, Thorstad WL, Lewis JS Jr. B7-H1 expression model for immune evasion in human papillomavirus-related oropharyngeal squamous cell carcinoma. Head Neck Pathol. 2013;7(2):113-21.

13 Majzner RG, Simon JS, Grosso JF, Martinez D, Pawel BR, Santi M, et al. Assessment of programmed death-ligand 1 expression and tumor-associated immune cells in pediatric cancer tissues. Cancer. 2017;123(19):3807-15.

14 Glinoer D. The medical treatment of endocrine exophthalmos. Bull Soc Belge Ophtalmol. 1988;226:59-64.

15 Haragan A, Field JK, Davies MPA, Escriu C, Gruver A, Gosney JR. Heterogeneity of PDL1 expression in non-small cell lung cancer: implications for specimen sampling in predicting treatment response. Lung Cancer. 2019;134:79-84.

16 McLaughlin J, Han G, Schalper KA, CarvajalHausdorf D, Pelekanou V, Rehman J, et al. Quantitative assessment of the heterogeneity of PD-L1 expression in non-small-cell lung cancer. JAMA Oncol. 2016;2(1):46-54.

17 Paintal AS, Brockstein BE. PD-L1 CPS scoring accuracy in small biopsies and aspirate cell blocks from patients with head and neck squamous cell carcinoma. Head Neck Pathol. 2020;14(3):657-65. 